\title{
A influência da alimentação saudável em crianças com Transtorno do Espectro Autista
} (TEA)

\author{
The influence of healthy eating in children with Autistic Spectrum Disorder (ASD) \\ La influencia de la alimentación saludable en niños con Trastorno del Espectro Autista (TEA)
}

Recebido: 30/10/2021 | Revisado: 07/11/2021 | Aceito: 11/11/2021 | Publicado: 20/11/2021

Marcely Viana Pavão

ORCID: https://orcid.org/0000-0003-3616-3824 Universidade Nilton Lins, Brasil

E-mail: marcely.pavao@gmail.com

Karen Celiane das Chagas Cardoso

ORCID: https://orcid.org/0000-0003-0926-4347

Universidade Nilton Lins, Brasil

E-mail: karenceliane@gmail.com

\begin{abstract}
Resumo
O objetivo geral da pesquisa é realizar uma revisão da literatura sobre a influência de uma alimentação saudável em crianças com transtorno do espectro autista (TEA). Aponta-se os seguintes objetivos específicos: definir e caracterizar o transtorno do espectro autista, compreender os principais problemas nutricionais das crianças com autismos; e evidenciar a importância da alimentação saudável em pessoas com transtorno autista. A metodologia usada neste estudo consiste em uma revisão narrativa, realizada através de levantado nas seguintes bases de dados: PubMed, SciELO, Google Acadêmico e biblioteca virtual de saúde (BVS), publicados no período de 2014 a 2021. Compreendeu-se que a sensibilidades alimentares desenvolvidas pelas crianças com TEA, como a seletividade alimentar, deficiência de ferro, e alergia alimentar afeta a ingestão nutricional adequadas, que influencia a saúde, comportamento e apresentação clínica do transtorno. Conclui-se que uma dieta sem glúten, caseína, e muita açúcar, porém com a adição de suplementos vitamínicos são formas muito positivas que ajudam a reduzir as alterações de comportamentos alimentares, bem como a uma diminuição do comportamento autoagressivo.
\end{abstract}

Palavras-chave: Transtorno do Espectro Autista; Alimentação saudável; Nutrição infantil.

\begin{abstract}
The general objective of the research is to carry out a literature review on the influence of healthy eating in children with autism spectrum disorder (ASD). The following specific objectives are pointed out: to define and characterize the autism spectrum disorder, to understand the main nutritional problems of children with autism; and highlight the importance of healthy eating in people with autistic disorder. The methodology used in this study consists of a narrative review, carried out through surveys in the following databases: PubMed, SciELO, Academic Google and virtual health library (VHL), published from 2014 to 2021. Foods developed by children with ASD, such as food selectivity, iron deficiency, and food allergy affect adequate nutritional intake, which influences the health, behavior, and clinical presentation of the disorder. It is concluded that a diet without gluten, casein, and lots of sugar, but with the addition of vitamin supplements are very positive ways that help to reduce changes in eating behaviors, as well as a decrease in self-aggressive behavior.
\end{abstract}

Keywords: Autistic Spectrum Disorder; Healthy eating; Child nutrition.

\section{Resumen}

El objetivo general de la investigación es realizar una revisión de la literatura sobre la influência de la alimentación saludable en niños con trastorno del espectro autista (TEA). Se señalan los siguientes objetivos específicos: definir y caracterizar el trastorno del espectro autista, comprender los principales problemas nutricionales de los niños con autismo; y destacar la importancia de una alimentación saludable en personas con trastorno autista. La metodología utilizada en este estudio consiste en una revisión narrativa, realizada a través de encuestas en las siguientes bases de datos: PubMed, SciELO, Academic Google y Virtual Health Library (BVS), publicadas entre 2014 y 2021. Alimentos desarrollados por niños con TEA, como la selectividad alimentaria, la deficiencia de hierro y la alergia alimentaria afectan la ingesta nutricional adecuada, lo que influye en la salud, el comportamiento y la presentación clínica del trastorno. Se concluye que una dieta sin gluten, caseína y mucha azúcar, pero con la adición de suplementos vitamínicos son formas muy positivas que ayudan a reducir los cambios en las conductas alimentarias, así como una disminución de las conductas autoagresivas.

Palabras clave: Trastorno del Espectro Autista; Alimentación saludable; Nutrición infantil. 


\section{Introdução}

Os problemas alimentares é uma realidade entre os autistas, o que afeta negativamente a qualidade de vida as crianças com autismo, assim surge a importância do acompanhamento nutricional desde cedo, para que seja elaborado um plano alimentar nutritivo e equilibrado (Bottan et al., 2020).

O transtorno do espectro autista (TEA) é uma patologia do neurodesenvolvimento de origem neurobiológica que afeta o desenvolvimento da comunicação social e comportamental. Apresenta uma evolução crônica, com diferentes graus de afetação, adaptação funcional e momento evolutivo (Mas, 2018). Na visão de Mapelli et al. (2018, p.2) "sua prevalência mundial é da ordem de 10/10.000 crianças, sendo superior nas do sexo masculino; para cada uma menina, cinco meninos são autistas".

Além disso, o TEA pode ser caracterizado por déficits persistentes na comunicação social entre contextos e pela presença de comportamentos, interesses e / ou atividades restritas e repetitivas (Petitpierre \& Luisier, 2021). Seus padrões alimentares apresentam rejeição dos alimentos/recusa ou preferências por determinados tipos de alimentos (Bottan et al., 2020). Outras apresentações clínicas alimentares do autismo, segundo Moreira (2019, p.3), são dificuldades na alimentação, característica típica da doença, pela rejeição ao novo e alteração da rotina alimentar. [...] Além disso, com tantas preocupações em relação ao tratamento adequado, a saúde bucal é quase sempre negligenciada. Por isso observa-se nestas crianças uma alimentação rica em alimentos doces, dificuldade na higienização e uso de medicamentos xerestomogênicos (risperidona, vitamina B6, haloperidol, fluoxetina, entre outros), os quais podem contribuir para uma saúde bucal precária.

Além disso, crianças com TEA apresentam problemas de comportamento alimentar como recusa alimentar, seletividade alimentar, sensibilidades sensoriais, ingestão calórica baixa, alimentação excessiva e comportamentos perturbadores durante as refeições (Chalane, 2021). Por isso, apresentam uma prevalência maior de sobrepeso e obesidade (Goularte et al., 2020).

O presente artigo analisa a correlação entre a nutrição e o TEA, tendo em vista fatores como a seletividade alimentar, uma vez que se compreende que a alimentação e a nutrição são importantes na gestão do autismo, justificando os cuidados dietéticos individualizados no momento do seu tratamento, pois cada criança com TEA tem uma combinação de anormalidades clínicas e laboratoriais que lhe são próprias. Uma apropriada intervenção dietética permite um maior alívio dos sintomas da doença, em conjunto com as terapias, sendo está a justificativa do artigo.

Diante do contexto apresentado, o objetivo geral da pesquisa é realizar uma revisão da literatura sobre a influência de uma alimentação saudável em crianças com transtorno do espectro autista (TEA). Aponta-se os seguintes objetivos específicos: definir e caracterizar o transtorno do espectro autista, compreender os principais problemas nutricionais das crianças com autismos; e evidenciar a importância da alimentação saudável em pessoas com transtorno autista.

\section{Metodologia}

Uma das etapas da revisão é a busca da literatura que visa descobrir se a questão da pesquisa foi respondida por outro estudo, ou até por outra revisão literatura do tipo narrativa. Para isso é realizado uma pesquisa de estudo elegíveis, devidamente selecionados. O objetivo é reunir o maior número de referências pertinentes, e para isso deve ser elaborado uma boa estratégia de busca (Donato \& Donato, 2019).

Entre os artigos de revisão, podemos também distinguir entre revisões narrativas. Uma revisão narrativa apresenta um relato da literatura no sentido de uma visão geral, incluindo tipos de literatura diferentes (pesquisa, relatórios do go verno, etc.).

Sendo assim, a base de dados para o estudo foram: Para busca das informações coletadas usou-se os seguintes termos: Transtorno do Espectro Autista, Alimentação Saudável, Nutrição Infantil; Locais da pesquisa foi realizada via internet nas bases de dados PubMed, Biblioteca Virtual de Saúde (BVS), SciELO, Google Acadêmico, e outras revistas que tratam sobre o tema proposto. 
Para seleção de artigos foram considerando critérios de inclusão e exclusão a estratégia elaborada para a inclusão nessa pesquisa foram: estudo publicados em revistas indexadas em inglês, espanhol e português, disponíveis na íntegra, gratuitos, que abordassem sobre o tema: "Transtorno do Espectro Autista", "Alimentação Saudável”, e "Nutrição Infantil". No qual foram devidamente publicados no período de 2014 a 2021. A estratégia elaborada para exclusão foram estudos publicados antes de 2015, que não estavam disponíveis na integra, e não tinham relação com o tema proposto.

\section{Resultados e Discussão}

\subsection{Definição e características do Autismo}

O TEA é uma condição relacionada ao desenvolvimento do cérebro que afeta a forma como uma pessoa percebe e socializa com os outros, causando problemas na interação social e comunicação. O distúrbio também inclui padrões de comportamento limitados e repetitivos (Magalhães, 2016).

Evidências científicas indicam causas multifatoriais, incluindo fatores genéticos e ambientais. Algumas crianças apresentam sinais de TEA no início da infância, como contato visual reduzido, falta de resposta ao nome ou indiferença aos cuidadores. Outras crianças podem desenvolver-se normalmente nos primeiros meses ou anos de vida, mas de repente ficam retraídas ou agressivas ou perdem habilidades de linguagem que já adquiriram. Os sinais geralmente são vistos aos 2 anos de idade (Mas, 2018).

No que diz respeito às questões motoras, são apresentados alguns movimentos estereotipados, ações atípicas repetitivas, além de dissimetrias na motricidade. No que concerne ao aspecto sensorial, algumas das ações comumente observadas envolvem a sensibilidade exagerada a determinados sons e insistência visual em objetos piscantes, que emitem barulhos e/ou giram (Chalane, 2021).

Já as rotinas, as pessoas autistas possuem a tendência de ser ritualizadas e rígidas, havendo também uma dificuldade nas modificações da alimentação, ao passo que, na fala, são apresentadas ações como a repetição de palavras que acabaram de ser ouvidas e a emissão de falas sem sentido contextual (Mel et al., 2021).

O diagnóstico do TEA é baseado em critérios clínicos com validação internacional e necessita de uma avaliação abrangente para ser realizado. É de grande importância a detecção precoce e o início de intervenções terapêuticas o mais cedo possível. A demora no diagnóstico e no tratamento pode acarretar prejuízo para o desenvolvimento da criança. A convicção errônea dos profissionais da saúde e dos pais de que se deve esperar o tempo da criança, mesmo quando esta apresenta atrasos nítidos no desenvolvimento, é um dos principais fatores que contribuiu para o atraso do diagnóstico e compromete o tratamento, desfavorecendo o prognóstico (Correia et al., 2011).

\subsection{Alimentação saudável na infância}

Comer de forma equilibrada e forma adequada é uma garantia de desenvolvimento em humanos, especialmente nas crianças e adolescentes, uma vez que a partir dos primeiros anos de vida, a dieta é um fator primordial para ter uma vida saudável ao longo dos anos (Ferreira, 2018).

De forma básica, pode-se explicar que as necessidades nutricionais são as quantidades de nutrientes essenciais mais a quantidade de energia que cada indivíduo precisa para atender a demanda que seu corpo requer. Entretanto, esses requisitos são variáveis para cada indivíduos, pois dependendo da idade, sexo, atividade física e condição fisiológicos (Gomes et al., 2016).

Uma alimentação saudável e equilibrada é essencial para o estado de saúde das crianças, sendo um fator determinante para um bom funcionamento do organismo, um bom crescimento, uma óptima capacidade de aprender, comunicar, pensar, socializar e adaptar-se a novos ambientes e pessoas, um correto desenvolvimento psicomotor e, em última instância, para a prevenção dos fatores de risco que influenciam no aparecimento de algumas doenças (Rosa, 2020). 
Uma criança com uma boa alimentação tem menos probabilidade de sofrer: distúrbios nutricionais, anemia, sobrepeso, obesidade, cárie dentária e problemas de aprendizagem escolar, contribuindo para a prevenção de certas patologias na idade adulta como doenças cardiovasculares, diabetes e alguns tipos de câncer (Rosa, 2020).

Para que essa dieta seja completa, todos os grupos de alimentos devem ser consumidos, incluindo carboidratos, frutas e vegetais, proteínas, laticínios, gorduras e açúcares (Coelho, 2019). A nutrição adequada beneficia as crianças que estão no estágio de crescimento, mas infelizmente muitas vezes é afetado por fatores socioeconômicos, falta de preparo nutricional dos pais e responsáveis ou uma dieta desequilibrada (Faria, Santos \& Vieira, 2021).

\subsection{Nutrição da criança com autismo}

Os pacientes com TEA podem apresentar distúrbios gastrointestinais como: dor abdominal, azia, bruxismo, perda de peso, irritabilidade, constipação, excesso de opioides cerebrais por alta absorção intestinal, alterações na permeabilidade da mucosa gástrica e defeitos enzimáticos, que podem ser a causa da chamada enteropatia artística. E estas apresentações clínicas, muitas vezes desconhecidas por seus responsáveis, impactam na alimentação das crianças com TEA (Arévalo Baque, 2018).

Além disso, os problemas metabólicos impedem o funcionamento normal do sistema digestivo, uma vez que pacientes com diagnóstico de TEA podem apresentar intestino mais permeável do que o resto da população, o que pode causar a incapacidade de metabolizar alguns aminoácidos essenciais, desencadeando fenilcetonúria, hiperuricemia, intolerância ao glúten e caseína (Loyacono et al., 2020). Caetano e Gurgel (2018, p. 8) completam que as crianças diagnosticas com TEA possuem as seguintes apresentações clínicas:

"Maior risco de excesso de peso, pois possuem grandes dificuldades em praticar exercícios físicos de forma
estruturada, além do isolamento social, o que possibilita o aumento de sedentarismo e corrobora os dados desta
pesquisa, em que todos os participantes não praticam nenhuma atividade fisica. Porém, o sobrepeso e a obesidade são
problemas de saúde pública na população em geral, pois a incidência de muitas doenças crônicas na vida adulta está
diretamente ligada à obesidade na infância".

Dificuldades alimentares experimentadas por pessoas com TEA também incluem rituais em torno das refeições, recusa alimentar, alimentação rápida, problemas de mastigação, dificuldade de adaptar o comportamento alimentar a outras pessoas presentes na situação alimentar, embolsar alimentos sem engolir por um período prolongado de tempo, enchendo a boca demais, vômitos e / ou ingestão de substâncias não alimentares (Petitpierre \& Luisier, 2021).

A seletividade alimentar da criança com TEA é o comportamento desordenado mais comumente relatado em crianças com TEA e é observada ao longo da vida. Apresentam preferência por carboidratos e aversão a frutas, grãos inteiros e vegetais. Aceitação e recusa de alimentos podem ser baseadas em sua cor, apresentação, cheiro, temperatura e textura (Smile, Raffaele \& Perlin, 2021).

Os fatores contribuintes para seletividade de alimentos em crianças com TEA são diversos, variando de experiências negativas de alimentação, constipação, doença do refluxo gastroesofágico, desafios motores orais e diferenças sensoriais. A recusa por alimentos saudáveis tem um impacto significativo na saúde e no bem-estar dessa população, uma vez que implica em deficiências nutricionais de ferro, baixos níveis de proteína e cálcio (Rocha et al., 2019).

A seletividade alimentar é um termo usado para se referir a variedade limitada e a ingestão restritiva de alguns alimentos comumente consumidos. Portanto, o diagnóstico precoce da patologia é muito importante, a fim de fazer uma intervenção nutricional precoce para evitar desequilíbrios nutricionais (Gama et al., 2020).

Os problemas relacionados a alimentação de crianças com TEA acarretam vários riscos para o equilíbrio de nutrientes e o crescimento da criança. Eles também podem limitar o prazer que as pessoas com TEA podem ter durante as refeições e fazer 
com que percam oportunidades de compartilhamento e participação social e de relaxar na companhia de outras pessoas, mesmo que alguns, principalmente adultos, desenvolvam estratégias para lidar com o social (Lázaro, Siquara \& Pondé, 2020).

A importância da alimentação é fundamental para o bom desenvolvimento físico e psicológico do paciente com TEA (Faria, Santos \& Vieira, 2021). Entretanto, compreende-se que cada criança diagnosticada com TEA possui diferentes necessidades, por isso a importância da dieta individualizada em função da necessidade distinta de cada um (Moura, Da Silva, Landim, 2021).

\subsection{A Influência a Influência da Alimentação Saudável em Crianças com TEA}

O TEA não é apenas caracterizado por distúrbios comportamentais e de comunicação, mas também pode haver problemas relacionados à nutrição, como obesidade, desejo por certos alimentos, deficiência imunológica, dor abdominal, azia, bruxismo, perda de peso, irritabilidade, constipação, hipoglicemia e problemas metabólicos (Caetano \& Gurgel, 2018). Em muitos casos, não recebe a importância que merece, causando crises, falta de sono, irritabilidade e outros efeitos negativos (Araruna et al. 2018).

A contribuição do nutricionista em TEA está relacionada às alterações no nível gastrointestinal e no comportamento em relação à alimentação, refletidas em seu estado nutricional e composição corporal. Indivíduos com TEA apresentam altas taxas de transtornos alimentares, com prevalência de sobrepeso / obesidade e, raramente, baixo peso (Felipe et al., 2021).

Ingestão inadequada de cálcio, ferro, vitamina B5, ácido fólico, vitamina C, magnésio, zinco e ácidos graxos essenciais, podem estar associados a distúrbios neurológicos, e uma dieta rica em carboidratos concentrados e ingestão de sódio são observados nesta população acima dos valores de referência (Araruna et al. 2018).

Uma ingestão adequada de vitamina B6 (piridoxina) é essencial, uma vez que ajuda o corpo a converter alimentos em glicose, que é usada para produzir energia e formar neurotransmissores, que carregam sinais de uma célula nervosa para outra; produzem hormônios, glóbulos vermelhos células do sistema imunológico; controle (junto com vitamina B12 vitamina B9) o nível de homocisteína no sangue, um aminoácido que pode estar associado a doenças cardíacas (Araruna et al. 2018; Gomes, 2020).

Estudos indicam que algumas dietas oferecem benefícios, segundo estudo realizado por Felipe et al. (2021) uma das dietas mais utilizadas pela população, e eliminar produtos com glúten (presente no trigo, cevada e centeio) e caseína (presente em laticínios e derivados). O glúten e a caseína têm sido associados à liberação de peptídeos com atividade opioide no intestino, que podem atravessar a mucosa intestinal.

Para Gomes et al. (2016, p.5) devido ao distúrbio gastrointestinal das crianças com TEA é recomendado a seguinte dieta:

"A dieta isenta de caseína e glúten é considerada uma alternativa segura para amenizar os sintomas gastrointestinais dos autistas, no entanto requer ao máximo de atenção dos nutricionistas, uma vez que é adotada por muitos indivíduos autistas. [...] a adequada abordagem nutricional, inserida num programa multidisciplinar de tratamento da doença constituiria uma mais-valia para os doentes, contribuindo para a melhoria da sua situação clínica e da sua capacidade funciona"l.

Devido a essas restrições, pode ocorrer uma ingestão inadequada nutrientes, o que leva ao desenvolvimento de doenças crônicas e degenerativas, que tendem a surgir na terceira ou quarta década de vida, ou ainda mais cedo, no caso de distúrbios menstruais (em meninas), apneia do sono e transtornos psíquicos (Araruna et al. 2018).

Nessa mesma linha de alimentação adequada da criança com TEA Rocha et al. (2019, p.2-3) realiza a seguinte afirmação: 
"Uma nutrição adequada ajuda na prevenção de doenças, no bom funcionamento do organismo, além de proporcionar uma melhor qualidade de vida. Para a manutenção de uma nutrição adequada é necessário que o indivíduo consuma uma variedade de alimentos, pois essa variedade traz uma maior oferta de nutrientes. Crianças com TEA podem apresentar dificuldades em aceitar novas experiências alimentares, o que pode ocasionar deficiência de algum nutriente".

Entretanto, o mais importante é uma boa dieta para crianças com diagnóstico de TEA é incluir proteínas, carboidratos, gorduras, vitaminas e sais minerais para alcançar uma boa ingestão nutricional em sua dieta, considerando as necessidades individuais. Por isso, todo o cardápio deve ser projetado de acordo com necessidades apresentadas pela criança levando em consideração sua idade, peso, altura, sexo e a atividade da criança (Gomes et al., 2016).

Diante disso, destaca-se a importância da avaliação nutricional, pois trata-se de um dos pilares fundamentais do manejo dos indivíduos com TEA. Porém destaca-se que abordagem deve ocorrer em conjunto com um grupo multidisciplinar, com o objetivo de melhorar a saúde de crianças com TEA. Assim, o nutricionista deve oferecer conhecimento os pais e responsáveis para que eles possam conduzir diariamente a dieta dessas crianças (Arévalo Baque, 2018).

\section{Conclusão}

O estudo alcançou seu objetivo porque compreendeu, por meio de uma revisão narrativa, a influência de uma alimentação saudável em crianças com TEA. Diante das conceituações expostas, compreendeu-se que a sensibilidades alimentares desenvolvidas pelas crianças com TEA, como a seletividade alimentar, deficiência de ferro, e alergia alimentar afeta a ingestão nutricional adequadas, que influencia a saúde, comportamento e apresentação clínica do transtorno.

Além disso, a pesquisa concluiu que uma dieta sem glúten, caseína, e muito açúcar, porém com a adição de suplementos vitamínicos são formas muito positivas que ajudam a reduzir as alterações de comportamentos alimentares, bem como a uma diminuição do comportamento autoagressivo, portanto, ajudando no transtorno e na conduta nesses tipos de crianças, promovendo a qualidade de vida delas.

Porém, devido às restrições alimentares, deve-se ter cuidado a ingestão inadequada de nutrientes, por isso é importante que tenham um acompanhamento nutricional para que o indivíduo consuma uma variedade de alimentos, pois essa variedade traz uma maior oferta de nutrientes.

Entretanto, destaca-se que a dieta deve ser adaptada às necessidades e condições individuais de cada criança com TEA, o mais recomendado é fazer uma alimentação saudável de acordo com o estado nutricional da criança em horários rígidos com baixo consumo de açúcar. Assim, o suporte nutricional e outras intervenções são importantes para vida, e não devem ser levados em consideração apenas na infância, mas ao longo do ciclo vital, pois mudanças satisfatórias são relatadas em qualquer momento da vida.

Em suma, compreende-se que o papel do acompanhamento nutricional é fundamental porque oferecer suporte adaptado as crianças com TEA com a finalidade de suprir suas necessidades nutricionais. Destaca-se que o profissional de nutrição oferecerá aos pais e responsáveis um cardápio diversificado com diferentes opções com finalidade de tornar a experiência de comer mais agradável e mais acessível.

\section{Agradecimentos}

Agradecemos a todos que direta ou indiretamente contribuíram para a realização e sucesso do artigo.

\section{Referências}

Arévalo Baque, E. S. (2018) Valoración nutricional en niños y adolescentes con trastorno del espectro autista en la Fundación Autismo Ecuador en el período mayo-septiembre Trabalhão de conclusão (Nutrição) - Universidade de Santiago; 
Araruna, L. L. (2018) Influência da alimentação no tratamento de crianças com transtorno do espectro do autismo. Faculdade de Ciências da Educação e Saúde Curso De Nutrição, Centro Universitário De Brasília - UniCEUB.

Bottan, G. P., Duarte, C. N., dos Santos Santana, J. R., Mendes, R. D. C. D., \& Schmitz, W. O. (2020). Analisar a alimentação de autistas por meio de revisão de literatura. Brazilian Journal of Development, 6(12), 100448-100470.

Caetano, M. V., \& Gurgel, D. C. (2018). Perfil nutricional de crianças portadoras do transtorno do espectro autista. Revista brasileira em promoçao da saúde, 31(1), 1-11.

Coelho, V. T. S. (2019). A formação de hábitos alimentares na primeira infância. Trabalho de Conclusão de Curso de Graduação em Nutrição do Centro Universitário Unifacvest.

Correia, T. L. B. V., Cunha, T. F. Q., Andrade, E. R. R., dos Santos, R. C., Maciel, E. A. F., Rezende, F. M., \& Pena, H. P. (2021). Alterações epigenéticas no transtorno do espectro autista: revisão integrativa de literatura. Research, Society and Development, 10 (11).

Gomes, V. T. S., Gomes, R. N. S., Gomes, M. S., Viana, L. V. M., Conceição, F. R., Amorim, L. M. M., \& Soares, E. L. (2016). Nutrição e autismo: reflexões sobre a alimentação do autista. Revista Univap, São José dos Campos, 22(40).

Chalane, C. (2021) Comportamento alimentar de crianças e adolescentes autistas: uma revisão integrativa. Monografia (Nutrição) - Centro Universitário AGES

Faria, L. C. M., Santos, A. C. F., \& Vieira, K. H. (2021). Avaliação dos hábitos alimentares de crianças com o Transtorno do Espectro Autista (TEA): um estudo de caso. Bionorte, 10(2), 149-154.

Ferreira, C. (2018). A importância de uma alimentação adequada na infância (Bachelor's thesis, Universidade Tecnológica Federal do Paraná).

Felipe, J. S., Carvalho, A. C. C., Lamounier, C. N., Hanna, G. M., Daia, I. C. G., de Oliveira, L. M., \& Moura, L. R. (2021). Relação entre o espectro autista e os transtornos alimentares. Brazilian Journal of Health Review, 4(1), 1310-1324.

Flick, U. (2012). Introdução à metodologia de pesquisa: um guia para iniciantes. Penso Editora.

Gama, B. T. B., Lobo, H. H. M., da Silva, A. K. T., \& Montenegro, K. S. (2020). Seletividade alimentar em crianças com Transtorno do Espectro Autista (TEA): uma revisão narrativa da literatura. Revista Artigos. Com, 17, e3916-e3916.

Gomes, T. A. S. (2021). Modulação nutricional no transtorno do espectro autista - um estudo de caso. Rev Bras Nutr Func; 46(81).

Goularte, L. M., de Moraes, L. S., de Souza Silva, E., Maieves, H. A., Borges, L. R., \& Bertacco, R. T. A. (2020). Transtorno do Espectro Autista (TEA) e hipersensibilidade alimentar: perfil nutricional e prevalência de sintomas gastrointestinais. Revista da Associação Brasileira de Nutrição-RASBRAN, 11(1), 4858.

Lázaro, C. P., Siquara, G. M., \& Pondé, M. P. (2020). Escala de Avaliação do Comportamento Alimentar no Transtorno do Espectro Autista: estudo de validação. Jornal Brasileiro de Psiquiatria, 68, 191-199.

Loyacono, N., Sanz, ML, Gerbi, MD, Martínez, LM, Ferreira, ML, \& Iermoli, R. (2020). Problemas gastrointestinales, nutricionales, endocrinológicos e de microbiota no trastorno del espectro autista. Arco. argent. Pediatr, e271-e277.

Mapelli, L. D., Barbieri, M. C., Castro, G. V. D. Z. B., Bonelli, M. A., Wernet, M., \& Dupas, G. (2018). Criança com transtorno do espectro autista: cuidado na perspectiva familiar. Escola Anna Nery, 22.

Mas, N. A. (2018). Transtorno do espectro autista-história da construção de um diagnóstico (Doctoral dissertation, Universidade de São Paulo).

Melo, A., Pereira, V. Y., Souza, C. O. F., Castro, R., Teodoro, G. S., Barreto, R., \& Rivero, L. (2021). Desenvolvimento de uma aplicação educativa para o ensino derotinas diárias e quebra de rotinas a crianças Autistas. RENOTE, 19(1), 166-175.

Moura, G. V., da Silva, R. R., \& Landim, L. A. D. S. R. (2021). Seletividade Alimentar Voltada Para Crianças Com Transtorno Do Espectro Autista (TEA): Uma Revisão Da Literatura. Revista Arquivos Científicos (IMMES), 4(1), 14-19.

Petitpierre, G., Luisier, A. C., \& Bensafi, M. (2021). Eating behavior in autism: senses as a window towards food acceptance. Current Opinion in Food Science, 41, 210-216.

Rocha, G. S. S., de Medeiros Júnior, F. C., Lima, N. D. P., da Silva Machado, A., Pereira, I. C., da Silva Lima, M., \& da Silva, H. A. C. (2019). Análise da seletividade alimentar de pessoas com Transtorno do Espectro Autista. Revista Eletrônica Acervo Saúde, (24), e538-e538.

Rosa, C. (2020) Comer, comer... é o melhor para poder crescer: um estudo dos fatores que influenciam o consumo alimentar infantil. Dissertação (mestrado) Universidade Regional do Noroeste do Estado do Rio Grande do Sul (Campus Ijuí). Desenvolvimento Regional.

Smile, S., Raffaele, C., \& Perlin, R. (2021). Re-imagining the physicians' role in the assessment of feeding challenges in children with autism spectrum disorder. Paediatrics \& Child Health, 26(2), e73-e77. 